OPEN ACCESS

Edited by:

Temel Tirkes,

Indiana University, United States

Reviewed by:

Yuming Jiang,

Stanford University, United States

Ying-Shi Sun,

Peking University Cancer Hospital,

China

*Correspondence:

Xin-Sheng Zhang

zhangxs85@sina.com

Xiang Li

doc_lixiang@163.com

Specialty section:

This article was submitted to

Cancer Imaging and

Image-directed Interventions,

a section of the journal

Frontiers in Oncology

Received: 20 October 2020

Accepted: 26 February 2021

Published: 18 March 2021

Citation:

Cao Q, Lai S-Y, Xu N, Lu Y, Chen S, Zhang $X$-S and LiX (2021) Computed

Tomography Features of Gastric

Cancer Patients With DNA

Mismatch Repair Deficiency.

Front. Oncol. 11:619439.

doi: 10.3389/fonc.2021.619439

\section{Computed Tomography Features of Gastric Cancer Patients With DNA Mismatch Repair Deficiency}

\author{
Qian Cao ${ }^{1}$, Sheng-Yuan Lai ${ }^{1}$, Nan $X u^{1}$, Yang $L u^{1}$, Shuai Chen ${ }^{1}$, Xin-Sheng Zhang ${ }^{2 *}$ \\ and Xiang $\mathrm{Li}^{1 *}$ \\ ${ }^{1}$ Radiology Department, Second Affiliated Hospital of Dalian Medical University, Dalian, China, ${ }^{2}$ General Surgery \\ Department, Second Affiliated Hospital of Dalian Medical University, Dalian, China
}

Objective: To explore the computed tomography (CT) features of gastric cancer (GC) patients with DNA mismatch repair deficiency (dMMR).

Materials and Methods: This study reviewed the clinical and CT features of GC patients with dMMR, confirmed by the postoperative results, between September 2017 and December 2019. The expression pattern of MMR major proteins (MLH1, MSH2, MSH6, and PMS2) in immunohistochemistry was used to confirm the MMR status in GC tissues. The correlation between pre-treatment CT features and MMR status was statistically analyzed.

Results: A total of 28 patients with GC were diagnosed as dMMR in our study, and 49 patients were MMR-proficient (pMMR). The tumor locations were significantly different between the dMMR and pMMR groups ( $p=0.006)$. The CT tumor thickness, CT long and short diameters of the largest lymph node, and the number of lymph nodes on CT of the dMMR group were significantly different from the pMMR group.

Conclusion: The dMMR GC exhibited a lower stomach location, smaller tumor thickness and lymph node diameter, and fewer lymph nodes on CT imaging.

Keywords: gastric cancer, computed tomography, mismatch repair deficiency, microsatellite instability, prognosis

\section{INTRODUCTION}

Gastric cancer (GC) or adenocarcinoma is one of the most common cancers and a common cause of cancer-related deaths worldwide $(1,2)$. GC is an aggressive disease, and many GC patients have locally advanced disease at presentation in China (3). The Cancer Genome Atlas has identified microsatellite instability (MSI) with or without DNA mismatch repair deficiency (dMMR) as a hallmark of the second molecular subtype of GC. Immunotherapy in solid malignant tumors, including GC, has been rapidly evolving. Immune checkpoint inhibitors, including antiprogrammed death-1 (PD-1) and anti-cytotoxic T-lymphocyte-associated protein-4 (CTLA-4) antibodies, were effective for MSI-high or dMMR solid tumors in many trials (4). However, the dMMR status often requires postoperative pathological immunohistochemical results or polymerase chain reaction testing. 
Multi-detector computed tomography (CT) is currently the routine modality of choice for preoperative examination of GC (5-10). CT can provide morphological information about primary tumors, lymph nodes, and suspected distant metastases. A previous report found that dMMR GC features included intestinal-type histology, antral location, and good prognosis with a low rate of recurrence (11). However, whether GC with dMMR has characteristic imaging findings on CT is unknown. In our study, we explored GC patients' CT features with dMMR for early and advanced GC.

\section{MATERIALS AND METHODS}

\section{Patients}

This retrospective study was approved by the review board of our institution. The requirement for informed consent was waived. We collected the clinicopathological data of patients with pathologically confirmed GC who underwent radical gastrectomy between September 2017 and December 2019 in our hospital. Some patients underwent two to three cycles of neoadjuvant chemotherapy. All patients underwent baseline contrast-enhanced CT examination of the abdomen. Patients were excluded if they met any of the following criteria: (a) They were detected with distant metastasis in the preoperative examination or during the operation. (b) Patients with poor quality CT images or those who did not undergo preoperative CT due to poor physical condition or other reasons.

Finally, 87 patients ( 65 males, 22 females, mean age, 58 years; range, 39-85 years) were included in our study, of which 39 patients received radical gastrectomy directly, and 48 patients underwent radical gastrectomy after neoadjuvant chemotherapy (NAC). A flowchart of the study design is presented in Figure 1.

\section{CT Protocol}

A CT scanner with 128 rows of detectors was used (Philips Brilliance iCT 256, Royal Dutch Philips Electronics Ltd, Amsterdam, Netherlands). After at least 8 hours of fasting, the patients were given $1,000 \mathrm{ml}$ water for achieving gastric distension. First, the non-contrast CT scan from the diaphragm's dome to $2 \mathrm{~cm}$ below the lower margin of the airdistended gastric body was acquired (collimation: $0.625 \mathrm{~mm}$, peak tube voltage: $120 \mathrm{kVp}$, tube current-time product: automatic). Next, $100 \mathrm{ml}$ of non-ionic contrast medium (Ultravist, $370 \mathrm{mg} / \mathrm{ml}$; Bayer, Germany) was intravenously administered at $3 \mathrm{ml} / \mathrm{s}$ using an automatic injector. Contrastenhanced CT scans were performed in the arterial phase (30 s) and the portal venous phase (70 s). The portal venous phase was used to evaluate lymph node status. The portal venous phase axial CT images were reconstructed with a $5-\mathrm{mm}$ section

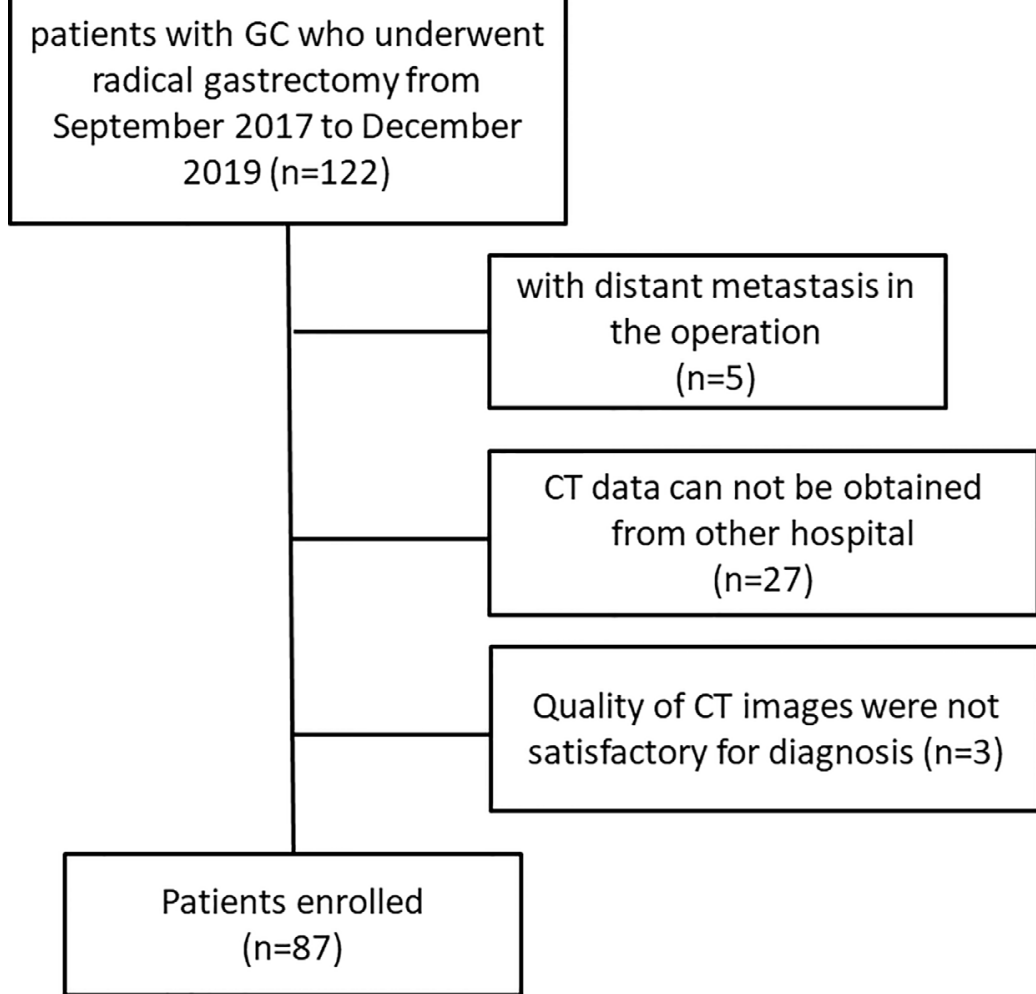

FIGURE 1 | Flowchart of the study design. 
thickness and a 5-mm reconstruction interval for clinical interpretation, with a $0.625-\mathrm{mm}$ section thickness for multi planar reformation (MPR) reconstruction.

\section{Image Analysis}

The image analysis was jointly performed by two radiologists with more than 10 years of experience, using the PACS workstation on the axial arterial phase and portal phase CT images. Any discrepancies were resolved by consulting another radiologist with 20 years of experience, and the consensus was achieved. The CT features of GC observed and measured were as follows:

Tumor Location: The location of GC in the CT images was determined by the radiologists, including esophagogastric junction, upper stomach, middle stomach, and lower stomach. We have made the correlation between CT images and endoscopy results to confirm the tumor's location for every case.

Tumor Thickness: The thickest diameter of the gastric tumor on the axial CT image was measured before and after NAC.

CT Attenuation of Gastric Tumor in Arterial and Portal Phases: The region of interest (ROI) was placed in the whole tumor center with a diameter $\geq 5 \mathrm{~mm}$. The $\mathrm{CT}$ attenuation values of the gastric tumor in the same portion of the axial arterial and portal phase CT images were measured.

Long and Short Diameters of the Largest Lymph Node: The largest regional lymph node's long and short diameters were measured on axial CT images.

CT Attenuation of the Largest Lymph Node: The CT attenuation values with an oval ROI of the largest regional lymph node on the axial portal phase CT image was measured.

The Number of Lymph Nodes: The number of all the short diameters of gastric regional lymph nodes $>5 \mathrm{~mm}$ in the axial portal phase images were counted.

\section{Pathological Diagnosis}

The postoperative histopathological diagnosis was performed by an experienced pathologist. The tumor in the gross specimen, the histopathological Lauren classification, and the pathological stage was evaluated based on the eighth AJCC Cancer Staging Manual (12). The expression patterns of MMR major proteins (MLH1, MSH2, MSH6, and PMS2) in immunohistochemistry were used to confirm the MMR status in GC tissues by the experienced pathologist. The lack of expression of any of the four MMR proteins was defined as dMMR. Tumors with the preserved expression of all MMR proteins were considered MMR-proficient (pMMR).

\section{Statistical Analysis}

The continuous and categorical data were presented as mean \pm standard deviation and frequency (\%). Data processing and analysis were performed using SPSS/PC+ version 22.0 (SPSS Inc, Chicago, IL, USA). The CT features of the dMMR and pMMR groups were compared using the independent-samples t-test and Mann-Whitney U test. A p-value $<0.05$ was considered statistically significant.

\section{RESULTS}

\section{Patient and Tumor Characteristics}

Eighty-seven patients were included in this study. The patient and tumor characteristics are summarized in Table 1. Among the patients, 48 received NAC before surgery. The NAC regimens included SOX (S-1 + oxaliplatin), XELOX (oxaliplatin + capecitabine), and mFOLFOX7 (modified regimen of leucovorin, fluorouracil, and oxaliplatin). The remaining 39 patients underwent surgery without NAC. The tumor locations were significantly different between the $\mathrm{dMMR}$ and pMMR groups $(\mathrm{p}=0.006)$. The age, gender, tumor size, histological differentiation degree, and pathological stage showed no statistical differences between the dMMR and pMMR groups ( $\mathrm{p}>0.05)$ (Table 1).

\section{Comparison of CT Features Between the dMMR and pMMR Groups of GC}

Univariate analysis showed that several CT features were significantly different between the dMMR and pMMR groups during surgery. The CT tumor thicknesses of the dMMR group $(11.89 \pm 4.87 \mathrm{~mm})$ were less than the pMMR group (14.41 \pm $4.70 \mathrm{~mm})(\mathrm{p}=0.024)$. The CT long diameters of the largest lymph node of the dMMR group $(8.71 \pm 2.43 \mathrm{~mm})$ were less than the pMMR group $(10.61 \pm 3.82 \mathrm{~mm})(\mathrm{p}=0.018)$. The CT short diameters of the largest lymph node of the dMMR group $(6.21 \pm$ $2.17 \mathrm{~mm})$ were less than the pMMR group $(7.44 \pm 2.85 \mathrm{~mm})(\mathrm{p}=$ 0.047). The mean number of lymph nodes on CT of the dMMR group $(1.71 \pm 1.41)$ was less than the pMMR group $(2.56 \pm$ $1.98 \mathrm{~mm})(\mathrm{p}=0.046)$ (Table 2). The CT attenuation of the gastric tumor and the largest lymph node after enhancement showed no significant differences between the dMMR and pMMR patients (Table 2).

\section{DISCUSSION}

A recent study found that anti-PD-1 therapy with pembrolizumab was clinically beneficial in patients with previously treated unresectable or metastatic MSI-H/dMMR non-colorectal cancer (13). In 2017, the Food And Drug Administration of the United States approved pembrolizumab to treat patients with dMMR/ MSI-H non-resectable or solid metastatic tumors. The MSI status is currently used as a biomarker for cancer immunotherapy (14). In our study, we examined some common CT features of the primary tumor and lymph nodes in patients with MSI-H/dMMR of GC.

Cristescu $\mathrm{R}$ et al. reported that dMMR GC typically has an antral location (11). The results of the present study showed that the tumor locations were significantly different between the dMMR group and the pMMR group. In our study, 53.5\% (15/ 28) dMMR patients were located at the lower stomach. Meanwhile, the main location of the pMMR group was the esophagogastric junction (57.6\%, 34/59) (Figures 2, 3). Good prognosis and low recurrence rate were thought to be more common in patients with dMMR GC. Although it has been recognized that the pathological stage was related to prognosis, the tumor size and pathological stage showed no statistical 
TABLE 1 | Patient characteristics.

\begin{tabular}{|c|c|c|c|}
\hline Clinicopathological features & dMMR (n = 28) & pMMR ( $n=59)$ & $\mathrm{p}$-value \\
\hline Male:female & 17:11 & $48: 11$ & 0.063 \\
\hline Tumor location & & & 0.006 \\
\hline Upper stomach & 2 & 3 & \\
\hline Middle stomach & 6 & 7 & \\
\hline Lower stomach & 15 & 15 & \\
\hline Medium differentiated adenocarcinoma & 4 & 21 & \\
\hline Mucinous adenocarcinoma & 0 & 1 & \\
\hline Neuroendocrine carcinoma & 1 & 2 & \\
\hline Pathological stage without NAC & & & 0.495 \\
\hline IA & 3 & 4 & \\
\hline IB & 3 & 1 & \\
\hline Pathological stage after NAC & & & 0.074 \\
\hline IA & 0 & 3 & \\
\hline IB & 2 & 2 & \\
\hline$\| A$ & 6 & 7 & \\
\hline$\| \mathrm{B}$ & 1 & 11 & \\
\hline IIIA & 1 & 7 & \\
\hline IIIB & 0 & 5 & \\
\hline IIIC & 0 & 3 & \\
\hline Tumor long size in the gross specimen $(\mathrm{cm})$ & $4.26 \pm 2.42$ & $4.09 \pm 2.40$ & 0.762 \\
\hline
\end{tabular}

${ }^{*} N A C$, neoadjuvant chemotherapy.

TABLE 2 | Comparison of CT image features between dMMR and pMMR patients.

\begin{tabular}{|c|c|c|c|c|}
\hline CT features* & dMMR (n = 28) & pMMR (n = 59) & $\mathbf{T}$ & $\mathrm{p}$-value \\
\hline Tumor thickness (mm) & $11.89 \pm 4.87$ & $14.41 \pm 4.70$ & 2.302 & 0.024 \\
\hline $\mathrm{CT}$ value of gastric tumor in $\mathrm{AP}(\mathrm{HU})$ & $70.78 \pm 27.86$ & $69.64 \pm 20.57$ & 0.215 & 0.083 \\
\hline CT value of gastric tumor in PP (HU) & $81.78 \pm 21.71$ & $84.25 \pm 24.64$ & 0.415 & 0.679 \\
\hline Long diameters of the largest LN (mm) & $8.71 \pm 2.43$ & $10.61 \pm 3.82$ & 2.402 & 0.018 \\
\hline Short diameters of the largest LN (mm) & $6.21 \pm 2.17$ & $7.44 \pm 2.85$ & 2.013 & 0.047 \\
\hline CT value of the largest LN in PP (HU) & $60.57 \pm 34.06$ & $59.41 \pm 28.08$ & 0.169 & 0.867 \\
\hline Number of lymph nodes on CT & $1.71 \pm 1.41$ & $2.56 \pm 1.98$ & 2.021 & 0.046 \\
\hline
\end{tabular}

${ }^{*} A P$, arterial phase; PP, portal phase; $L N$, lymph node.

differences between the dMMR and pMMR GC patients in our study.

Given that stomach is a hollow organ, the evaluation of tumor size in the stomach is often influenced by gastric peristalsis. Since the most common gross morphological type of advanced GC was the infiltrating ulcerative type, the boundaries of the tumor on CT images were difficult to identify clearly. In our study, each patient was given $1,000 \mathrm{ml}$ of water to achieve gastric distension. The tumor's thickness on CT seemed more suitable for evaluating tumor size. The CT tumor thickness of the dMMR group was less than the pMMR group. The CT long and short diameters of the largest lymph node of the dMMR group was less than the pMMR group in our study. Fukuya $\mathrm{T}$ et al. found that $\mathrm{CT}$ attenuation and lymph-node short-long size ratio could aid in the diagnosis of malignant adenopathy (15). Park et al. considered lymph nodes to be metastatic if the longest diameter was $>1.0 \mathrm{~cm}$ or if the size was between 0.7 and $1.0 \mathrm{~cm}$ with hyper-enhancement, a round shape, central necrosis, or perinodal infiltration (16). We thought that smaller diameters of the lymph node in the dMMR group indicated fewer lymph node metastases and a better prognosis. XP Zhang et al. reported that the number of lymph nodes detected by MDCT showed a significant difference between the lymph node metastasis group and no metastasis group in GC (17). The mean number of lymph nodes on CT in the dMMR group was less than the pMMR group in our study. This may suggest a lower probability of lymph node metastasis in the dMMR group.

This study had some limitations. First, it was a retrospective study. The sample size of the study was relatively small. 


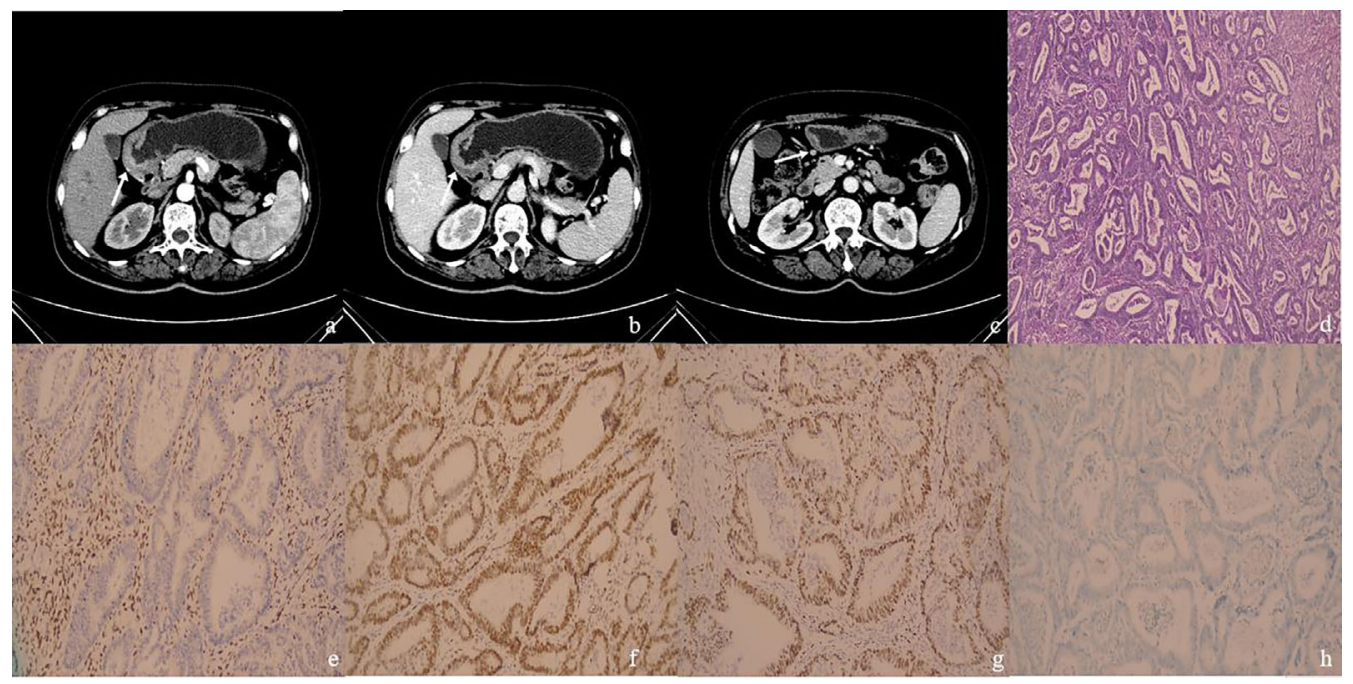

FIGURE 2 | (A-H): One MSI-H\&dMMR GC case. Female patient, 64 years old. The postoperative pathologic results showed moderately differentiated adenocarcinoma in gastric antrum with stage T3NOMO, with no metastatic carcinoma in lymph nodes. (A) The arterial phase CT value in the enhanced arterial phase of the thickened gastric wall (arrow) in the gastric antrum was $48 \mathrm{HU}$. (B) The portal phase CT value of the thickened gastric wall (arrow) was $67 \mathrm{HU}$. (C) There was a slightly enlarged lymph node (arrow) in No. 4d group around the stomach, with a short diameter of $5 \mathrm{~mm}$ and CT value of $72 \mathrm{HU}$ on the portal phase. (D) The case of histological analyses by HE staining. (E-H) The patient's immunohistochemical results showed MLH1-negative (E), MSH2-positive (F), MSH6-positive (G), PMS2negative (H), MSI-H\&dMMR.

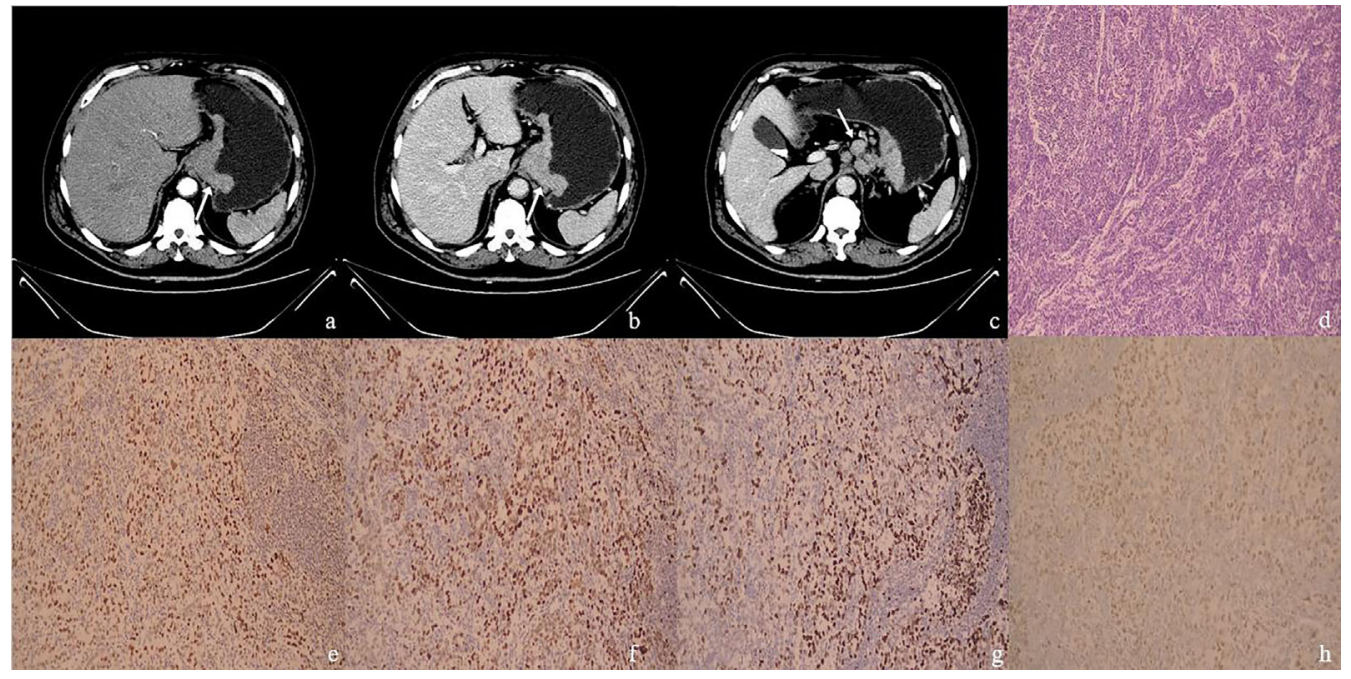

FIGURE 3 | (A-H) One MSS\&pMMR GC case. Male, 66 years old, surgical pathology results revealed poorly differentiated adenocarcinoma in the gastroesophageal junction, staging T4aN2M0, with metastatic carcinoma in lymph nodes. (A) The arterial phase CT value of the mass (arrow) in the gastroesophageal junction was 52 HU; (B) The portal phase CT value of the mass (arrow) was $82 \mathrm{HU}$; (C) Multiple enlarged lymph nodes could be seen in the stomach's lesser curvature. The largest one had a short diameter of $15 \mathrm{~mm}$ (arrow), with the CT value of $88 \mathrm{HU}$ on the portal phase. (D) The case of histological analyses by HE staining. (E-H) The immunohistochemical results showed MLH1-positive (E), MSH2-positive (F), MSH6-positive (G), PMS2-positive (H), MSS, pMMR.

The findings need to be confirmed by large prospective studies in the future. Second, in addition to MMR status, immunotherapy biomarkers of GC, including tumor mutation burden (TMB) and PD-L1 expression, were not analyzed in our study. Simultaneously, there is no confirmed study on whether neoadjuvant chemotherapy might affect patients' MMR status, which might require further research.

In summary, this study found that the dMMR GC exhibited a lower stomach location, smaller tumor thickness and lymph node diameter, and fewer lymph nodes on CT imaging. 


\section{DATA AVAILABILITY STATEMENT}

The raw data supporting the conclusions of this article will be made available by the authors, without undue reservation.

\section{ETHICS STATEMENT}

Ethical review and approval was not required for the study on human participants in accordance with the local legislation and institutional requirements. Written informed consent for participation was not required for this study in accordance with the national legislation and the institutional requirements.

\section{REFERENCES}

1. Siegel RL, Miller KD, Jemal A. Cancer statistics, 2018. CA Cancer J Clin (2018) 68(1):7-30. doi: 10.3322/caac.21442

2. Bray F, Ferlay J, Soerjomataram I, Siegel RL, Torre LA, Jemal A. Global cancer statistics 2018: GLOBOCAN estimates of incidence and mortality worldwide for 36 cancers in 185 countries. CA Cancer J Clin (2018) 68(6):394-424. doi: 10.3322/ caac. 21492

3. Yang L, Zheng R, Wang N, Yuan Y, Liu S, Li H, et al. Incidence and mortality of stomach cancer in China, 2014. Chin J Cancer Res (2018) 30(3):291-8. doi: 10.21147/j.issn.1000-9604.2018.03.01

4. Le DT, Uram JN, Wang H, Bartlett BR, Kemberling H, Eyring AD, et al. PD-1 Blockade in Tumors with Mismatch-Repair Deficiency. N Engl J Med (2015) 372 (26):2509-20. doi: 10.1056/NEJMoa1500596

5. Kim AY, Kim HJ, Ha HK. Gastric cancer by multidetector row CT: preoperative staging. Abdom Imag (2005) 30(4):465-72. doi: 10.1007/s00261-004-0273-5

6. Kwee RM, Kwee TC. Imaging in assessing lymph node status in gastric cancer. Gastric Cancer (2009) 12(1):6-22. doi: 10.1007/s10120-008-0492-5

7. Kumano S, Murakami T, Kim T, Hori M, Iannaccone R, Nakata S, et al. T staging of gastric cancer: role of multi-detector row CT. Radiology (2005) 237(3):961-6. doi: 10.1148/radiol.2373041380

8. Lim JS, Yun MJ, Kim MJ, Hyung WJ, Park MS, Choi JY, et al. CT and PET in stomach cancer: preoperative staging and monitoring of response to therapy. Radiographics (2006) 26(1):143-56. doi: 10.1148/rg.261055078

9. Kim JW, Shin SS, Heo SH, Choi YD, Lim HS, Park YK, et al. Diagnostic performance of 64-section CT using CT gastrography in preoperative $\mathrm{T}$ staging of gastric cancer according to 7th edition of AJCC cancer staging manual. Eur Radiol (2012) 22(3):654-62. doi: 10.1007/s00330-011-2283-3

10. Lee IJ, Lee JM, Kim SH, Shin CI, Lee JY, Kim SH, et al. Diagnostic performance of 64-channel multidetector CT in the evaluation of gastric cancer: differentiation of mucosal cancer (T1a) from submucosal involvement (T1b and T2). Radiology (2010) 255(3):805-14. doi: 10.1148/radiol.10091313

11. Cristescu R, Lee J, Nebozhyn M, Kim KM, Ting JC, Wong SS, et al. Molecular analysis of gastric cancer identifies subtypes associated with distinct clinical outcomes. Nat Med (2015) 21(5):449-56. doi: 10.1038/nm.3850

\section{AUTHOR CONTRIBUTIONS}

Conceptualization: X-SZ and XL. Data curation: QC and S-YL. Formal analysis: QC and NX. Funding acquisition: XL. Methodology: QC, YL, and SC. Supervision: X-SZ and XL. Validation: QC, S-YL, and XL. Writing: QC and S-YL. All authors contributed to the article and approved the submitted version.

\section{FUNDING}

This work was supported by Liaoning Provincial Natural Science Foundation of China (Grant Nos. 2019-ZD-0645).

12. Oh SE, An JY, Choi MG, Lee JH, Sohn TS, Bae JM. Comparisons of remnant primary, residual, and recurrent gastric cancer and applicability of the 8th AJCC TNM classification for remnant gastric cancer staging. Eur J Surg Oncol (2020) 20:S0748-983(20)30557-6. doi: 10.1016/j.ejso. 2020.06.032

13. Marabelle A, Le DT, Ascierto PA, Di Giacomo AM, De Jesus-Acosta A, Delord JP, et al. Efficacy of Pembrolizumab in Patients With Noncolorectal High Microsatellite Instability/Mismatch Repair-Deficient Cancer: Results From the Phase II KEYNOTE-158 Study. J Clin Oncol (2020) 38(1):1-10. doi: 10.1200/JCO.19.02105

14. Mizrahi J, Pant S. Immunotherapy in Gastrointestinal Malignancies. Adv Exp Med Biol (2020) 1244:93-106. doi: 10.1007/978-3-030-41008-7_5

15. Fukuya T, Honda H, Hayashi T, Kaneko K, Tateshi Y, Ro T, et al. Lymphnode metastases: efficacy for detection with helical CT in patients with gastric cancer. Radiology (1995) 197(3):705-11. doi: 10.1148/radiology.197. 3.7480743

16. Park SR, Lee JS, Kim CG, Kim HK, Kook MC, Kim YW, et al. Endoscopic ultrasound and computed tomography in restaging and predicting prognosis after neoadjuvant chemotherapy in patients with locally advanced gastric cancer. Cancer (2008) 112(11):2368-76. doi: 10.1002/cncr.23483

17. Zhang XP, Wang ZL, Tang L, Sun YS, Cao K, Gao Y. Support vector machine model for diagnosis of lymph node metastasis in gastric cancer with multidetector computed tomography: a preliminary study. BMC Cancer (2011) 11:10. doi: 10.1186/1471-2407-11-10

Conflict of Interest: The authors declare that the research was conducted in the absence of any commercial or financial relationships that could be construed as a potential conflict of interest.

Copyright (C) 2021 Cao, Lai, Xu, Lu, Chen, Zhang and Li. This is an open-access article distributed under the terms of the Creative Commons Attribution License (CC BY). The use, distribution or reproduction in other forums is permitted, provided the original author(s) and the copyright owner(s) are credited and that the original publication in this journal is cited, in accordance with accepted academic practice. No use, distribution or reproduction is permitted which does not comply with these terms. 